\title{
EDITORIAL
}

\section{UNITED IN A GROUP, EACH NURSE CAN BE EMPOWERED}

\author{
Julie Benbenishty \\ Faculty of Nursing, School of Medicine, Hadassah Hebrew University, Jerusalem, Israel
}

The COVID-19 pandemic has placed nurses at the epicentre of worldwide importance, forcing them to face individual, personal, family, and moral dilemmas, as they are afraid of transmitting the virus to loved ones, parents, and children, and of leaving dependent relatives alone while working not only normal hours but also overtime. In addition to these personal dilemmas and conflicts, they also experience social clashes, such as being regarded as national heroes, fighting the lifesaving battle every day, and remaining physically distanced from friends and contacts. Whom do we lean on for support? How do we cope and get up every day and return to the nursing battlefield?

In Israel, the national Society of Cardiac and Intensive Care Nursing is an organization which supports frontline ICU nurses. During the pandemic, the group played the role of disseminating daily COVID-19 statistics, facilitating transferring clinical treatment protocols from bedside nurse to hospital leaders, providing a platform for clinical decisionmaking, having discussions regarding family visiting policies, and collaborating to support each other. We all knew what each one of us was going through. We used the group's platform as a way to exchange ideas and as a sounding board in instances when we thought something was not right. Frequently, a circumstance within the group required objective perspectives to find a solution. For example, at the very onset of the pandemic, nursing care of the deceased was very controversial. Some countries burned the bodies or buried the deceased in common graves. A bedside nurse, facing end-of-life care for her first COVID-19 deceased patient, did not know what to do. A frantic digital social media message
(WhatsApp) was sent to all ICU nurses connected to the group. Within a minute, one nurse answered that her hospital had a cultural, medically acceptable authorized care protocol, which she immediately disseminated throughout the country.

Very quickly, the group initiated routine monthly online meetings, conferences, scientific journal club exchange, and a national research study. Training courses were exchanged and trainers volunteered to educate each other.

We need to promote, expand, and use our national professional societies. Not only are professional societies a group of individuals with a common goal, a shared language, and a deep understanding of what each member does every day in the workplace, but they also hold each other sacred. This shared orientation toward one's professional work follows an ethic of uniting to be and to become more human and humane - a model that honours the inner subjective experience of patients and practitioners alike (Watson, 2006).

Together, as a group, each nurse is empowered.

\section{Assoc. Prof. Julie Benbenishty, PhD, RN Academic consultant} e-mail: julie@hadassah.org.il

\section{References}

Watson, J. (2006). Caring theory as an ethical guide to administrative and clinical practices. Nursing Administration Quarterly, 30(1), 48-55. https://doi.org/10.1097/00006216$\underline{\text { 200601000-00008 }}$ 